\title{
Medical professionalism research characteristics and hotspots: a 10-year bibliometric analysis of publications from 2010 to 2019
}

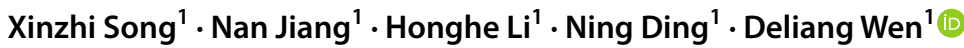

Received: 30 January 2021 / Accepted: 3 April 2021 / Published online: 5 July 2021

(c) Akadémiai Kiadó, Budapest, Hungary 2021

\begin{abstract}
An abundance of publications on medical professionalism have been published in recent years. Our study aimed to analyze the external characteristics of publications on professionalism using bibliometrics, to describe its current developments and trends, and to explore professionalism research hotspots using co-word analysis. We retrieved literature on professionalism published from 2010 to 2019 from the Web of Science database. Histcite and CiteSpace were used to analyze external characteristics of publications, including publication trends and distributions of publications by country/region, institution, author, and journal. Co-word analysis was conducted to analyze research hotspots, using the Bibliographic Item Co-Occurrence Matrix Builder and Graphical Clustering Toolkit. We constructed a strategic diagram to show the state of each research hotspot and the relationship between the various hotspots in this field. Results showed publications on professionalism increased in number year by year as a whole. The USA, including its institutions and authors, maintained the top position worldwide in terms of numbers of publications and citations. Among scholarly journals, Academic Medicine has published more articles and has had higher citations per paper than any other journal. Six research hotspots on professionalism were identified, visualized, and expounded. Of these, the theme of "measurement and evaluation of clinical competence" received the most attention in the field of professionalism. The reporting of quantifiable knowledge on the characteristics and research hotspots of publications could help inform gaps in the field of medical professionalism and provide evidence and guidance for future work for researchers, physicians, and educators.
\end{abstract}

Keywords Medical professionalism · Bibliometrics $\cdot$ Research hotspots $\cdot$ Co-word analysis $\cdot$ Biclustering

\section{Abbreviations}

ACGME Accreditation Council for Graduate Medical Education

ABIM American Board of Internal Medicine

EFIM European Federation of Internal Medicine

Deliang Wen

dlwen@cmu.edu.cn

1 Institute for International Health Professions Education and Research, China Medical University, No. 77 Puhe Road, Shenyang 110122, People's Republic of China 


$\begin{array}{ll}\text { GMC } & \text { General Medical Council } \\ \text { CMDA } & \text { Chinese Medical Doctor Association } \\ \text { RCPSC } & \text { Royal College of Physicians and Surgeons of Canada } \\ \text { SCIE } & \text { Science Citation Index-Expanded } \\ \text { SSCI } & \text { Social Science Citation Index } \\ \text { WOS } & \text { Web of Science } \\ \text { ESI } & \text { Essential Science Indicators } \\ \text { MeSH } & \text { Medical Subject Headings } \\ \text { BICOMB } & \text { Bibliographic Item Co-Occurrence Matrix Builder } \\ \text { gCLUTO } & \text { Graphical Clustering Toolkit }\end{array}$

\section{Introduction}

Medical professionalism influences doctor-patient relationships, patient satisfaction, quality of care, and even patient safety, and is therefore considered essential to medical practice (Barnhoorn et al., 2019; Lesser et al., 2010; Ong et al., 2020). An abundance of publications on professionalism have been published in recent years, and the topic of professionalism has also attracted extensive attention from medical organizations and professional societies across the globe. The Accreditation Council for Graduate Medical Education (ACGME), the American Board of Internal Medicine (ABIM), the European Federation of Internal Medicine (EFIM), UK's General Medical Council (GMC), and the Chinese Medical Doctor Association (CMDA) have all successively set out requirements for medical professionalism (Accreditation Council for Graduate Medical Education, 2004; Chinese Medical Doctor Association, 2014; General Medical Council, 2013; ABIM Foundation et al., 2002). The Royal College of Physicians and Surgeons of Canada (RCPSC) also described professionalism to be a key competency of the physician in the CanMEDs framework, with the professional being one of the seven CanMEDs roles (Frank et al., 2015). Due to variations across sociocultural, contextual, and geopolitical settings, professionalism has been considered to be a broad and complex concept (Hodges et al., 2011). Understanding current publication trends and hotspots would help physicians and medical educators track the development and variation of professionalism research and provide reference for future high-quality work on the research and practice of professionalism. Therefore, we made use of bibliometric methods to help identify external characteristics and research hotspots of publications on medical professionalism over the last decade.

Bibliometrics is a research method using quantitative statistical analysis to describe the external characteristics of publications and to evaluate and predict development trends in a particular research field (Pritchard, 1969). While research questions tend to be more narrow and focused in systematic reviews, which have generally centered on a precisely defined sub-topic in the field of professionalism, such as defining (Aylott et al., 2019; Birden et al., 2014; Dart et al., 2019), nurturing (Bergeret al., 2020; Birden et al., 2013; Brennan et al., 2020; Kumar Ghosh \& Kumar, 2019; Ong et al., 2020), or assessing professionalism (Hodges et al., , 2011, 2019; Li et al., 2017), bibliometrics focus on the metrological characteristics of a large number of papers published in a particular field (Ellegaard \& Wallin, 2015). Co-word analysis, an important bibliometric method developed by Michel Callon et al. (1986), has been widely used to identify research hotspots by analyzing the co-occurrence relationship between two professional terms in the same paper. Co-word analysis can 
be achieved by constructing a co-word matrix and then performing cluster analysis, eventually displaying research hotspots in the form of visualizations.

Although bibliometrics have been widely used and guidelines have been written in disciplines such as management (Linnenluecke, 2019), education (Diem \& Wolter, 2013), and information science (Michael, 2010), it has been rarely applied in the field of medical professionalism. Two previous studies have used bibliometrics to analyze publications on professionalism (Azer \& Azer, 2019; Hodges et al., 2019). Samy A. Azer and Sarah Azer analyzed the external characteristics and research topics of 50 top-cited articles published between 1994 and 2011 on professionalism (Azer \& Azer, 2019). Hodges et al. identified all publications on assessment of professionalism since 2011 and analyzed the publications by type, language, country, and primary epistemic domain of the collected texts (Hodges et al., 2019). These studies greatly contributed to the understanding of impact and trends in professionalism, but they were also limited in comprehensiveness by the number of articles and by focusing on a single aspect of professionalism.

Our study sought to achieve two primary purposes: (1) to analyze the external characteristics of publications in the field of medical professionalism, and (2) to explore research hotspots in medical professionalism using co-word analysis.

\section{Methods}

\section{Data sources and search strategies}

In order to obtain citation data for publications on medical professionalism and to perform bibliometric analyses, we used the Science Citation Index-Expanded (SCIE) and the Social Science Citation Index (SSCI) of the Web of Science (WOS) Core Collection as our data source, owing in particular to how it provides best-in-class publication and comprehensive citation data for many different scientific and social sciences disciplines (Clarivate, 2021). Our search strategy was based on strategies described in previously published systematic reviews of medical professionalism (Berger et al., 2020; Birden et al., 2013; Li et al., 2017). The detailed search strategy can be found in "Appendix A". Two authors with medical informatics background (X Song and $\mathrm{H} \mathrm{Li}$ ) independently conducted the primary search and reached consensus on the final search results. To avoid bias caused by frequent updates of databases, all searches and data downloads were completed within the same day on October 25, 2020.

\section{Eligibility criteria for publication selection}

Retrieved papers needed to meet the following inclusion criteria: (1) the publication year was between 2010 and 2019; (2) the publication language was in English; and (3) the document type was either an article or review.

\section{Analysis of distribution characteristics of publications}

For the first part, we described the external characteristics of publications, including publication trends, global geographic distributions of publications, and distribution of countries/regions, institutions, authors, and journals ranked by the number of publications. All 
countries/regions, institutions, and authors associated with a paper were considered to have made equal contributions to the paper (Tscharntkeet al., 2007), in congruence with the counting method for publications and citations used by the Essential Science Indicators (ESI) (Clarivate, 2020).

We used HistCite V12.03.17 to analyze the number of publications and the total and average citations of these publications by country/region, institution, author, and journal (Clarivate, 2016). We extracted the high-frequency keywords of authors with the most publications to get a glimpse of these authors' research fields. By aggregating publications and citations, we would be able to measure the level of scientific effort and prestige attributable to affiliated researchers at the national and institutional level (Clarivate, 2020). We calculated Pearson's correlation coefficient using SPSS V.24 to explore the potential correlation between Journal Impact Factor (JIF) in 2019 and citations per paper of these journals. A two-tailed $p$ value $<0.05$ was considered to be statistically significant.

We also used CiteSpace V5.7.R2 to calculate the betweenness centrality of every country/region and institution in collaboration networks (co-authorship networks). In collaboration networks, every node represented a country/region or institution. Betweenness centrality can be used to determine the importance of these nodes in the network and to reflect the degree of cooperation (Chen, 2003). Greater value of betweenness centrality meant greater importance the node has in the network and direct closer cooperation with other nodes (Chen, 2014).

\section{Analysis of research hotspots}

In the analysis of research hotspots, we first took into account and referenced Medical Subject Headings (MeSH), because they effectively avoid multiple words expressing the same concept and would thus present a better clustering result. We matched the papers retrieved from the WOS database with their corresponding paper in the PubMed database using their PMIDs, and then we used the Bibliographic Item Co-Occurrence Matrix Builder V2.02 (BICOMB) to extract all MeSH terms from these enrolled publications (Cui 2008; School of Medical Informatics of China Medical University, 2008).

To investigate the evolution of medical professionalism and co-topics, we identified $\mathrm{MeSH}$ terms related to the topic of medical professionalism based on the search strategy, including those MeSH terms and their subordinate terms corresponding to the search term that occurred in publications more than 5 times a year on average. We then analyzed the frequency of the MeSH term "Professionalism" and its co-topics published over time and tracked their patterns of growth and decline.

We performed co-word analysis of high-frequency MeSH terms to identify research hotspots in the field of professionalism. Pao's formula was used to calculate the threshold of high-frequency MeSH terms (T value): $\mathrm{T}=(-1+\sqrt{1+8 * I}) / 2(\mathrm{Pao}, 2014)$, within which $I$ represents the number of MeSH terms occurring only once. The MeSH terms with a frequency greater than or equal to the $\mathrm{T}$ value were defined as high-frequency MeSH terms. After that, we constructed a co-occurrence matrix of high-frequency MeSH terms using BICOMB and imported it into the Graphical Clustering Toolkit V1.0 (gCLUTO) to perform visualized clustering of the high-frequency MeSH terms (Rasmussen et al., 2004). Finally, we created a visual co-word matrix and mountain visualization, from which we determined research hotspots in the field of medical professionalism based on the highfrequency MeSH terms clusterings. 
Based on the clustering results of high-frequency MeSH terms, we used Microsoft Excel to construct a strategic diagram (Law et al., 1988) in order to show the state of each research hotspot and the relationship between the various hotspots in medical professionalism. The $x$-axis/y-axis of the strategic diagram represented the centrality/density of the cluster, respectively. Centrality (inter-cluster correlation) describes the intensity of connection of a given cluster with other clusters. Clusters with greater centrality are more likely to represent a set of research topics that the scientific community would consider crucial (Callon, 1991). Density (intra-cluster correlation) characterizes the strength of connection between the terms within the cluster. Clusters with greater density are more likely to contain research topics that would make up a coherent and integrated unit. The measure of density would provide representation of the cluster's sustainability and its ability to develop in the field under consideration over the course of time (Callon, 1991).

\section{Results}

\section{Distribution characteristics of publications}

Following our search strategy, a total of 5613 studies between 2010 and 2019 were retrieved from the initial search. Based on eligibility criteria, 340 studies were excluded due to publication language (not English), and 620 studies were excluded due to document type (not article or review). A total of 4653 publications were enrolled in the final study, comprising 4221 articles and 432 reviews.

\section{Global geographical distribution and temporal trend of publications}

Figure 1 shows the color-coded world map of the geographic distribution of publications from 2010 to 2019. A total of 104 countries/regions contributed to the 4653 publications. Among these 104 countries/regions, the majority of them are located in North

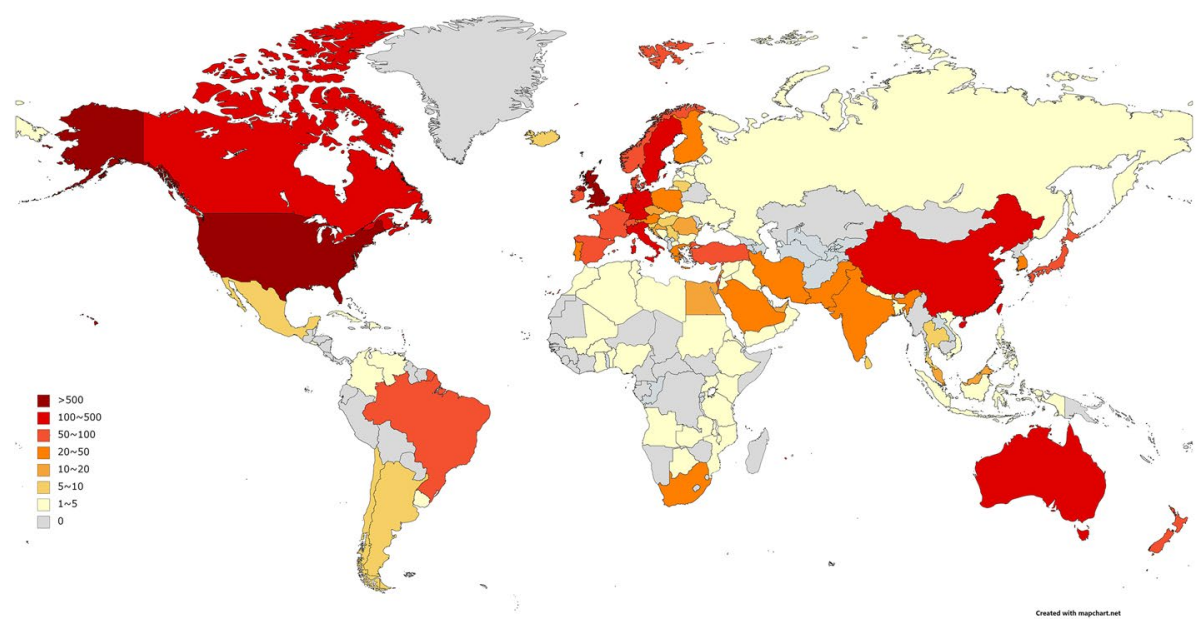

Fig. 1 Global geographic distribution of publications on medical professionalism between 2010 and 2019 
America (USA, Canada, etc.) and Europe (UK, Netherlands, etc.), while less than 15\% of the research results were from Asia (PRC, Japan, etc.) and Oceania (Australia, New Zealand, etc.). Table 1 shows the top 10 most productive countries that have contributed to scientific literature on medical professionalism. Among them, the United States contributed $49.56 \%$ of all publications. It also topped the list for most citations, accounting for $44.21 \%$ of the whole, followed by the United Kingdom, Canada, Australia, and Netherlands. The United States also scored highest in terms of centrality. Canada was the only country with a higher percentage of citations than publications and with the highest citations per paper. Figure 2 shows the publication trends and the number of annual publications from the top 5 countries compared with all other countries. As a whole, the number of publications showed an increasing trend year by year, increasing from 332 in 2010 to 681 in 2019-an average annual growth rate of $91.66 \%$. The top 50 countries/regions, institutions, authors (with top 3 most cited papers), and journals with the most publications/citations on medical professionalism can be found in "Appendix B".

\section{Contributions by institutions and authors to the global number of publications}

More than 4000 institutions and 16,000 authors contributed to work on medical professionalism research between the years 2010 and 2019. Tables 2 and 3 respectively show the top 10 institutions and top 10 authors with the most publications. All top 10 institutions were from the United States. Harvard University was the institution with the most publications (140) and citations (4762) and also occupied the core position in the network by having the highest centrality (0.16). Mayo Clinic was the institution with the highest citations per paper (36.61). Of all the top 10 authors, only two are from the United Kingdom, affiliated with Cardiff University and University of Dundee. Two of the most published authors (Eloy JA and Svider PF) are both from Rutgers New Jersey Medical School and have a close cooperative relationship; their publications mainly focused on malpractice and legal liability in various specializations.

Table 1 Top 10 countries/regions with the most publications on medical professionalism from 2010 to 2019

\begin{tabular}{llcccc}
\hline Ranking & Country/region & Publications, $N(\%)$ & Citations, $N(\%)$ & $\begin{array}{l}\text { Citations per } \\
\text { paper }\end{array}$ & $\begin{array}{l}\text { Between- } \\
\text { ness central- } \\
\text { ity }\end{array}$ \\
\hline 1 & & & & 0.29 \\
2 & USA & $2306(49.56)$ & $35,596(44.21)$ & 15.44 & 0.22 \\
3 & UK & $538(11.56)$ & $8421(10.46)$ & 15.65 & 0.21 \\
4 & Canada & $404(8.68)$ & $7732(9.60)$ & 19.14 & 0.12 \\
5 & Australia & $284(6.10)$ & $3678(4.57)$ & 12.95 & 0.07 \\
6 & Netherlands & $223(4.79)$ & $2990(3.71)$ & 13.41 & 0.02 \\
7 & PRC & $179(3.85)$ & $1070(2.10)$ & 9.46 & 0.07 \\
8 & Germany & $170(3.65)$ & $2613(3.25)$ & 15.37 & 0.09 \\
9 & Italy & $153(3.29)$ & $1723(2.14)$ & 11.26 & 0.05 \\
10 & Sweden & $104(2.24)$ & $999(1.24)$ & 9.61 & 0.04 \\
\hline
\end{tabular}




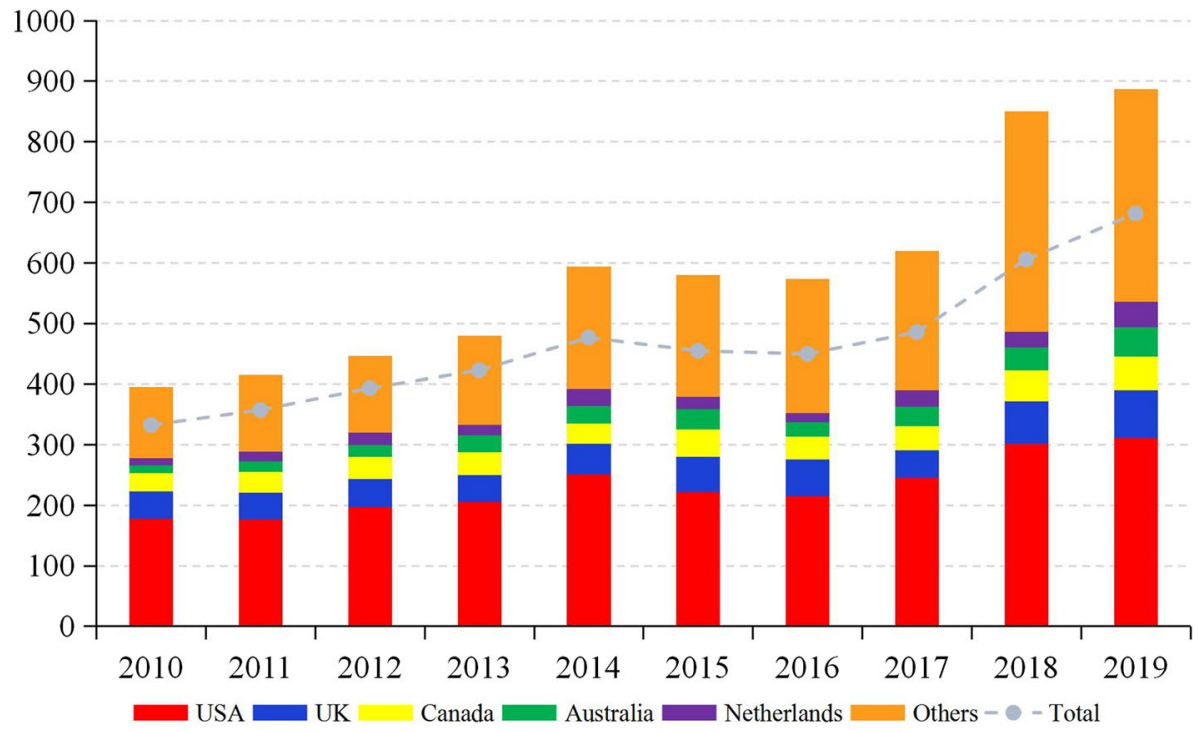

Fig. 2 Distribution of annual publications on medical professionalism between 2010 and 2019. The broken line graph shows the trend of total annual publications, and the stacked histogram shows the annual publications of the top 5 countries and other countries. All unique countries and regions were on a paper are equally credited, thus accounting for bigger graph

Table 2 Top 10 institutions with the most publications on medical professionalism from 2010 to 2019

\begin{tabular}{llcccc}
\hline Ranking & Institution & Publications, $N(\%)$ & Citations, $N(\%)$ & $\begin{array}{l}\text { Citations } \\
\text { per paper }\end{array}$ & $\begin{array}{l}\text { Between- } \\
\text { ness central- } \\
\text { ity }\end{array}$ \\
\hline 1 & & & $4762(2.57)$ & 34.01 & 0.14 \\
2 & Harvard Univ & $140(3.01)$ & $1954(1.05)$ & 19.16 & 0.05 \\
3 & Univ Washington & $102(2.19)$ & $1886(1.02)$ & 18.86 & 0.04 \\
4 & Univ Michigan & $100(2.15)$ & $3624(1.96)$ & 36.61 & 0.09 \\
5 & Mayo Clin & $99(2.13)$ & $1736(0.94)$ & 17.54 & 0.07 \\
6 & Univ Calif San Francisco & $99(2.13)$ & $2149(1.16)$ & 21.71 & 0.10 \\
7 & Univ Toronto & $99(2.13)$ & $1528(0.82)$ & 18.86 & 0.04 \\
8 & Johns Hopkins Univ & $81(1.74)$ & $666(0.36)$ & 8.22 & 0.02 \\
9 & Harvard Med Sch & $81(1.74)$ & $1954(1.05)$ & 27.52 & 0.03 \\
10 & Massachusetts Gen Hosp & $71(1.53)$ & $1175(0.63)$ & 18.65 & 0.04 \\
\hline
\end{tabular}

\section{Journals publishing research on medical professionalism}

All enrolled publications were produced by 1156 journals. These journals were classified as medical education journals and other journals, including general medical journals and specialty journals(Lee et al., 2013). Table 4 shows the top 10 journals with the most publications on medical professionalism. These 10 journals published more than $20 \%$ of the total (1015/4653). Academic Medicine and BMC Medical Education were the most 


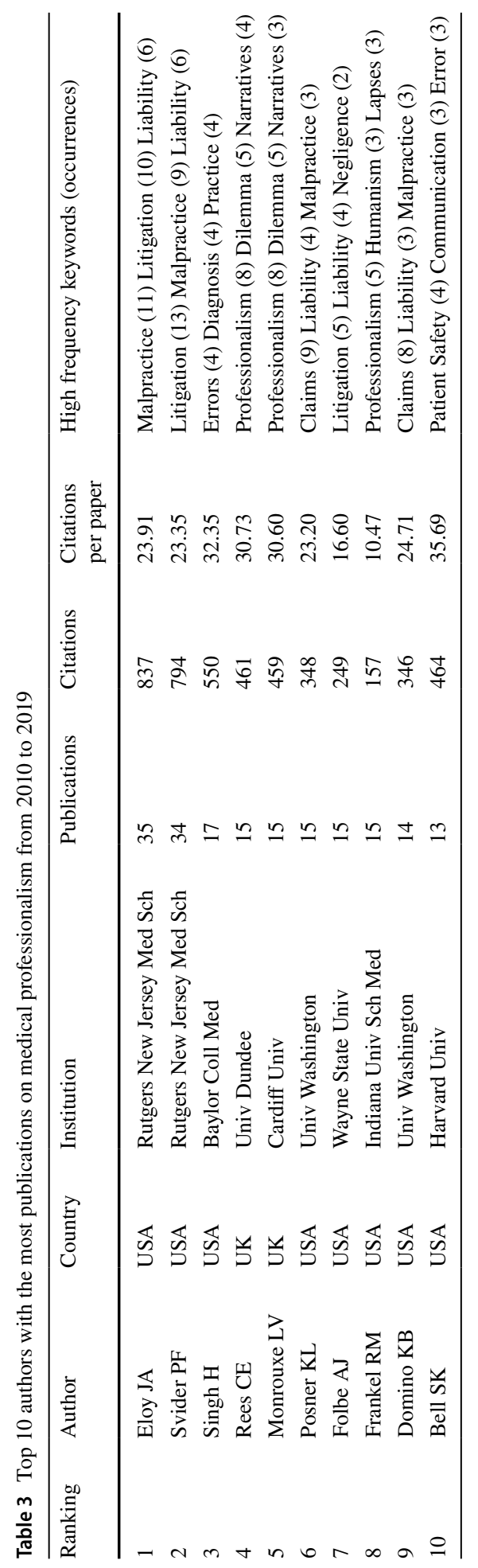


Table 4 Top 10 scholarly journals with the most publications on medical professionalism from 2010 to 2019

\begin{tabular}{|c|c|c|c|c|c|}
\hline Ranking & Journal & Publications, $N(\%)$ & Citations, $N(\%)$ & $\begin{array}{l}\text { Citations } \\
\text { per paper }\end{array}$ & JIF (2019) \\
\hline 1 & Academic Medicine & $219(4.71)$ & $7625(12.43)$ & 34.82 & 5.354 \\
\hline 2 & BMC Medical Education & $179(3.85)$ & $1690(2.75)$ & 9.44 & 1.831 \\
\hline 3 & Medical Teacher & $146(3.14)$ & $2084(3.40)$ & 14.27 & 2.654 \\
\hline 4 & Medical Education & $83(1.78)$ & $1930(3.15)$ & 23.25 & 4.57 \\
\hline 5 & Journal of Surgical Education & $71(1.53)$ & $765(1.25)$ & 10.77 & 2.22 \\
\hline 6 & BMJ Open & $71(1.53)$ & $563(0.92)$ & 7.93 & 2.496 \\
\hline 7 & $\begin{array}{l}\text { Teaching and Learning in } \\
\text { Medicine }\end{array}$ & $67(1.44)$ & $694(1.13)$ & 10.36 & 1.848 \\
\hline 8 & Journal of Medical Ethics & $63(1.35)$ & $489(0.80)$ & 7.76 & 2.021 \\
\hline 9 & PLoS One & $58(1.25)$ & $874(1.42)$ & 15.07 & 2.74 \\
\hline 10 & BMC Health Services Research & $58(1.25)$ & $607(0.99)$ & 10.47 & 1.987 \\
\hline
\end{tabular}

productive journals (highest number of publications), and Academic Medicine and Medical Education were the most influential journals (highest number of citations per paper) with regards to professionalism. Journal impact factor (JIF) in 2019 was positively associated with citations per paper among the top 10 journals with the most publications, based on a Pearson's correlation coefficient of $0.958(p<0.01)$.

\section{Research hotspots}

\section{Evolution of medical professionalism and co-topics}

Evolution of medical professionalism and co-topics, represented by MeSH terms related to the topic of professionalism based on the search strategy, are shown in Table 5 and Fig. 3. Professionalism was introduced into the MeSH database in 2016 (U.S. National Library of Medicine, 2016). Therefore, no paper was indexed as "Professionalism" from 2010 to 2013, but, owing to a delay in indexing of MeSH terms, a handful of papers were indexed as "Professionalism" from 2014 to 2015. Since 2016, the number of papers indexed with "Professionalism" had rapidly grown. "Professional Role" showed a similar development trend to "Professionalism", with fluctuating increase from 2010 to 2019. There was no significant trend for other MeSH terms.

\section{Research themes belonging to high-frequency MeSH terms clustering}

The 4653 papers from the WOS database were matched to 4348 papers in PubMed by means of PMID, with a match rate of $93.45 \%$. From these 4348 papers, we extracted 2213 major MeSH terms, among which 999 occurred only once $(I=999)$. Using Pao's formula, the threshold of high-frequency major MeSH terms was defined as 45 $(T=45)$. We selected the top 40 high-frequency major MeSH terms for co-word biclustering analysis. Ultimately, six clusters were created, with clusters 0 and 1 exhibiting 


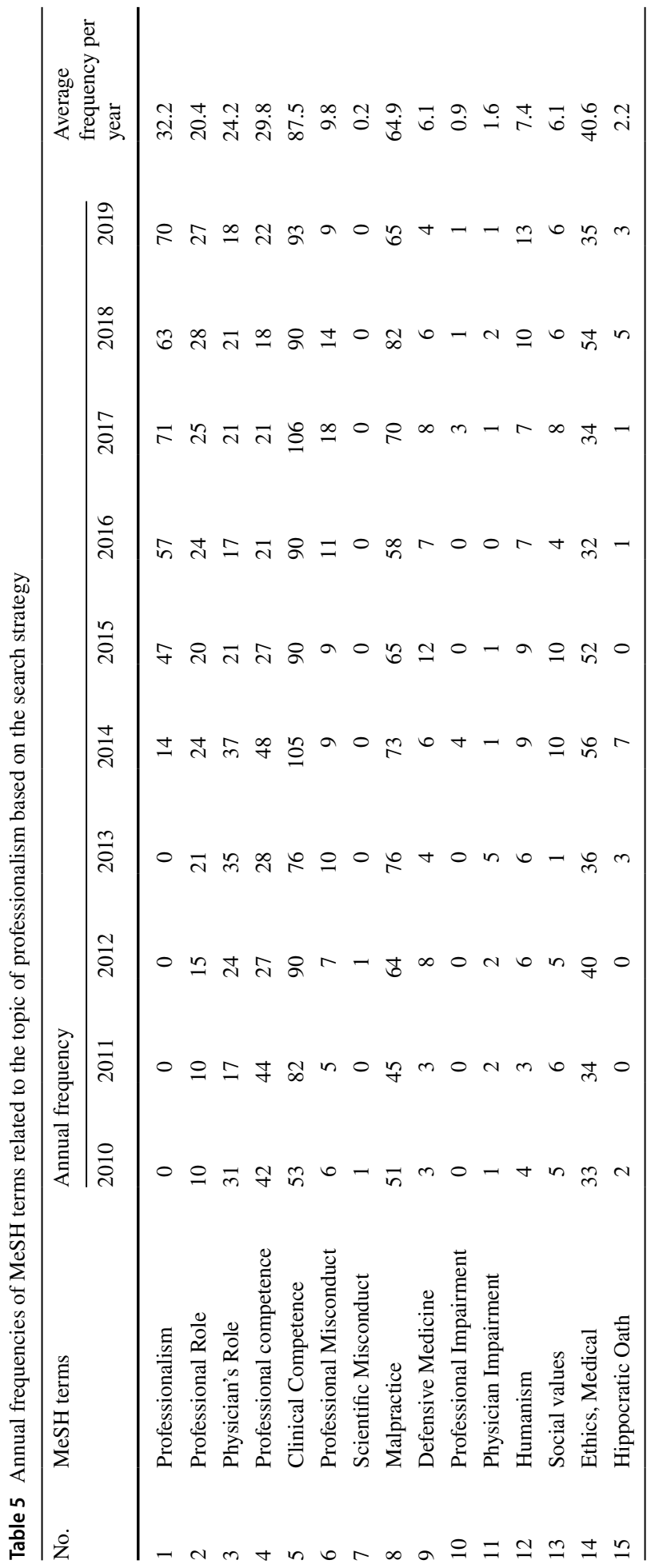



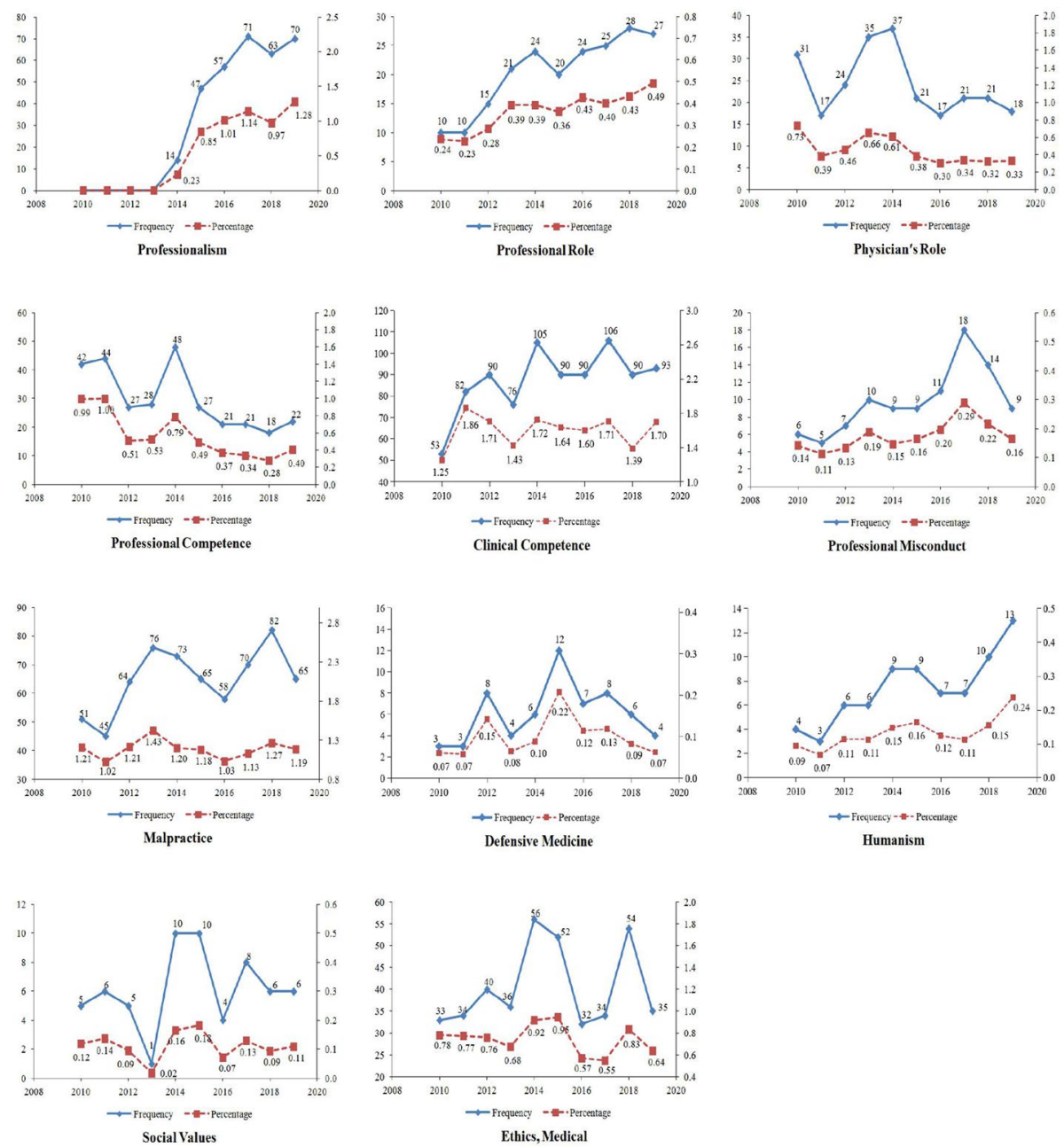

Fig. 3 Evolution of professionalism and co-topics, represented by MeSH terms related to the topic of professionalism based on the search strategy

the highest intra-cluster correlation and cluster 2 exhibiting the lowest standard deviation values. Major MeSH terms occurring within the same cluster indicated that these terms co-occurred more frequently. The research theme of each cluster were identified based on the terms in the cluster and the representative papers (by PMID) provided by gCLUTO in its solution section. The emerging themes were: (1) malpractice, negative healthcare outcomes, and corresponding solutions (Cluster 0); (2) measurement and evaluation of clinical competence (Cluster 1); (3) climates of professionalism (including e-professionalism) and unprofessional behaviors (Cluster 2); (4) teaching and learning in medical education, especially medical ethics (Cluster 3); (5) the professional role and interprofessional experiences of healthcare professionals (Cluster 4); and (6) positive 


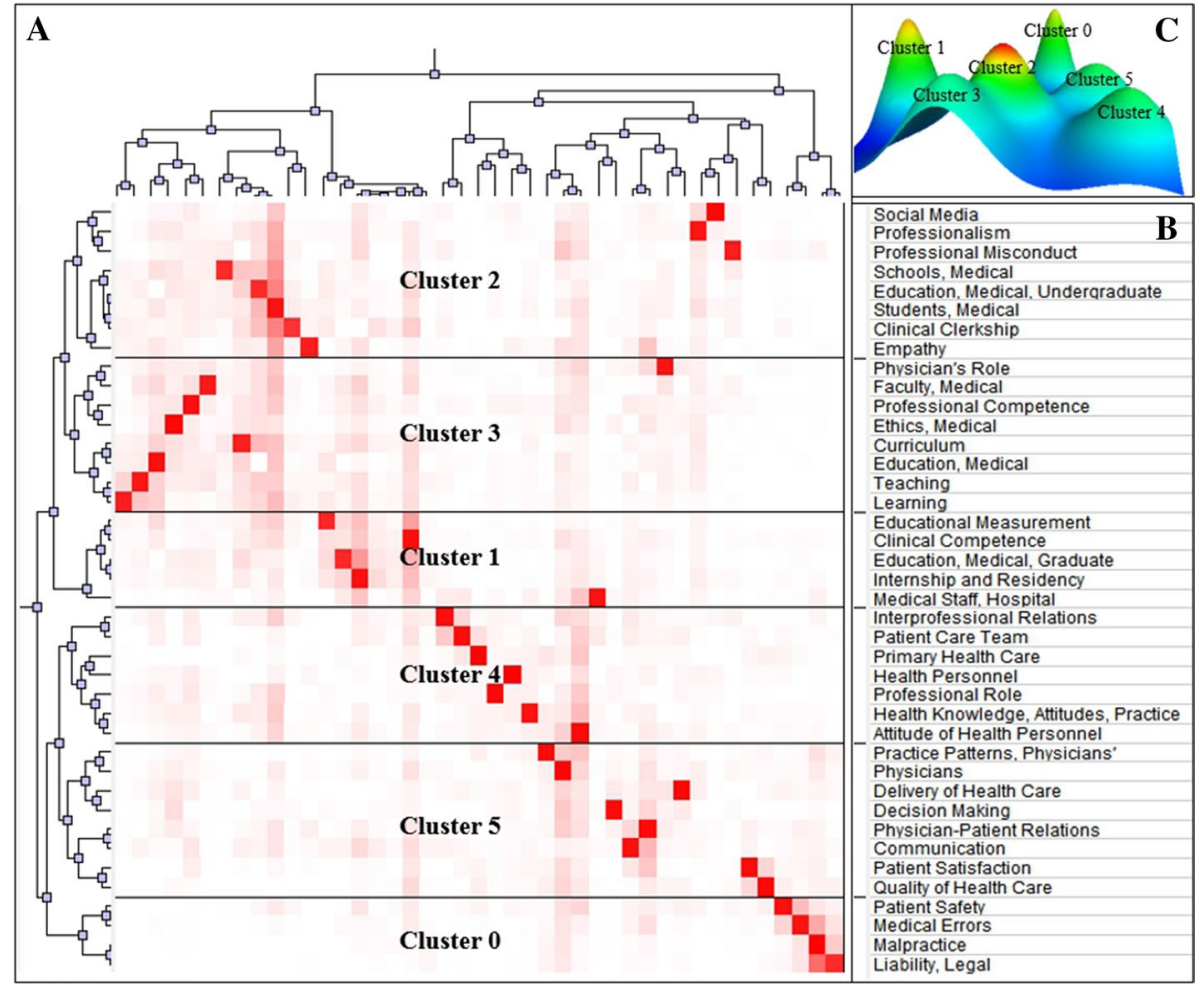

Fig. 4 Visual co-occurrence matrix (a), 40 high-frequency major MeSH terms (b), and cluster mountain map (c). Both the $x$-axis and $y$-axis in the co-occurrence matrix (a) represent the 40 high-frequency major $\mathrm{MeSH}$ terms (b). The row labels from left to right are the same as the column labels from top to bottom, and the red block represents the co-occurrence between two corresponding terms. The height of each peak in (C) represents the internal similarity of the corresponding cluster. The volume of each peak represents the number of included major MeSH terms. The color of a peak represents the internal standard deviation of the cluster's objects. Red, yellow, green, and blue represent deviation from low to high. The distance between a pair of peaks on the plane represents the relative similarity of their clusters. Clusters that are more similar will have peaks that lie closer together

factors to physician-patient relationships and quality of health care (Cluster 5). The visual co-occurrence matrix and mountain visualization are shown in Fig. 4.

\section{Strategic diagram}

The strategic diagram was constructed based on the high-frequency major MeSH terms clustering results (Fig. 5). Cluster 1 not only showed high intra-cluster correlation but also high inter-cluster correlation, thereby being considered as the core theme of professionalism research. Cluster 0 exhibited good intra-cluster correlation, but it was least relevant to the other clusters. Clusters 2, 3, 4 and 5 were closely connected with other clusters, but the correlations among $\mathrm{MeSH}$ terms within each of these clusters were poor. 


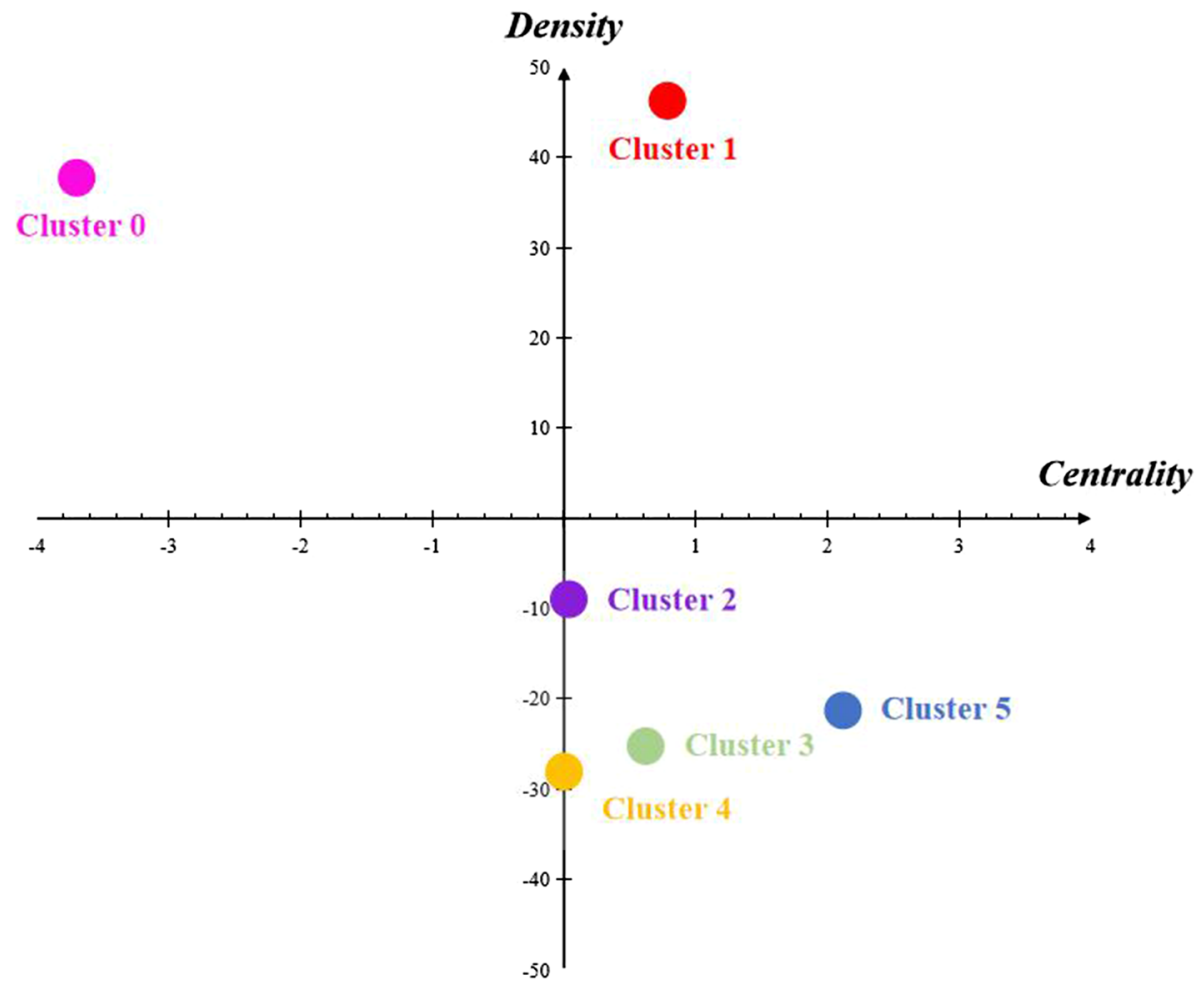

Fig. 5 The strategic diagram of research themes on medical professionalism by density and centrality. Clusters in the first quadrant (e.g., Cluster 1) are both central to the general network (they are strongly connected to other clusters) and have strong internal density (they display a high degree of development). These clusters in a sense constitute the core. Their position is strategical, and they have most likely been dealt with systematically over a long period by a well-defined group of researchers. Clusters in the second quadrant (e.g., Cluster 0) are not central—we will call them peripheral—and the strength of their internal links leads us to suppose that they correspond to research problems whose study has already been well-developed. They appear as specializations that interact weakly with the other sub-networks of the field under study. Clusters in the third quadrant (no cluster was found to belong to this quadrant) are both peripheral and underdeveloped. They represent the margins of the network. Only a dynamic analysis of the evolution of a network over several periods or a comparative analysis of the relationship of the network with other networks would allow us to determine their contribution to the field. Clusters in the fourth quadrant (e.g., Cluster 2, 3, 4 and 5) are central, indicating that they are strongly connected to other clusters, but the densities of their internal connections are relatively low

\section{Discussion}

In the present study, we illustrated the rapid growth of research publications on medical professionalism over the past 10 years, with annual publications increasing from 332 in 2010 to 681 in 2019 . Using co-word analysis, we identified and visualized six research hotspots on professionalism and then expounded on these research hotspots in combination with the strategic diagram.

Publications were widely distributed among North American and European nations. A large portion of publications came from the United States, where both its institutions and its authors have made great contributions to research in professionalism. This 
does not come as a surprise, since professional societies in the USA have been exploring professionalism from decades prior (Mueller, 2009). Some notable examples include the "Physician Charter", jointly issued by the ABIM (ABIM Foundation et al., 2002) and the core competencies laid out by the ACGME, in which professionalism was listed as one of six core competencies that residents must possess (Accreditation Council for Graduate Medical Education, 2004). The proportion of publications from other countries around the world also increased annually, suggesting a gradual increase in global interest in the field of medical professionalism.

We found that medical education journals have become an important source of research on professionalism. For example, the top 5 scholarly journals with the most publications are all medical education journals. In addition, we provided a long-term (10-year) citation analysis for journals on medical professionalism and compared this with short-term citation behaviors (2019 JIF), and found an internal consistent correlation between these two figures.

The strategic diagram illustrates the correlations between clusters of professionalism-related co-topics. In recent years, "measurement and evaluation for clinical competence" (Cluster 1) has become the core hotspot in the field of professionalism, with highest density and high centrality. According to Stufflebeam, the most important purpose of program evaluation is not to prove but to improve (Madauset al., 1983). Themes in this cluster focus on improving medical staffs' clinical competence and promoting their professional development. In accordance with the framework for conceptualizing professional behaviors by Lesser et al. (2010), achieving self-improvement in clinical competence for healthcare professionals is an important manifestation of the pursuit of excellence. In this framework, the pursuit of excellence includes "Interactions With Patients and Family Members" (e.g., adhering to nationally recognized evidence-based treatment guidelines) and "Interactions With Colleagues and Other Members of the Health Care Team" (e.g., participating in collaborative efforts to improve system-level factors contributing to quality of care). In addition to the professionalism behaviors framework by Lesser et al., both the ACGME and the ABIM listed "(commitment to) excellence" as an attribute when defining professionalism. (American Board of Internal Medicine 1995; Mueller, 2009).

Cluster 0 had high density but the lowest centrality. Differing from other clusters, Cluster 0 focused on negative outcomes of health care. The aftermath of medical errors, mostly involving physician negligence, often plays out through malpractice suits (Diederich Healthcare, 2017; US Department of Health and Human Services, 2017). Malpractice increases the risk of medical errors and thus threatens the safety of patients (Gómez-Durán et al., 2013; Kravet et al., 2019). By clarifying the accountability and responsibility of physicians, the possibility of malpractice can be reduced, and the occurrence of medical errors can be prevented and controlled, forming a benign chain reaction. In fact, physicians may exhibit behavior of different levels throughout their career, such as exemplary behaviors, professional behaviors, professional misconduct (unprofessional behaviors), and malpractice (professional negligence). Although professional misconduct and malpractice both influence the quality of health care, they generally cause different outcomes. "Professional misconduct" was indexed as "malpractice" prior to 2001. In 2002, "professional misconduct" became its own separate MeSH term. Professional misconduct refers to the "violation of laws, regulations, or professional standards" (U.S. National Library of Medicine, 2002) but are unlikely to cause medical errors, whether harmful or non-harmful. Malpractice, on the other hand, poses a serious threat to patient safety and is defined as the "failure of a physician to render proper services through reprehensible ignorance or negligence or 
through criminal intent, especially when injury or loss follows" (U.S. National Library of Medicine, 1974).

Cluster 2 described the climate of professionalism (including e-professionalism) and unprofessional behaviors during clinical clerkship. The professionalism climate in the clinical environment, which manifests through the hidden curriculum, may impact empathy and shape attitudes and behaviors (both professional or unprofessional) (Aziz \& Ali, 2020). In the three dominant professionalism frameworks described by Irby et al. (2016), the discussion of professionalism constructs, educational strategies, and remediating professionalism lapses all contribute to a professionalism climate for medical students and professionals. These frameworks shed light on the development of medical students into qualified doctors. To help guide medical students' and physicians' social media behaviors, some researchers have also proposed a series of principles for e-professionalism (Borgmann et al., 2018; Ellaway et al., 2015; Fatollahi et al., 2020). In addition, researchers from different cultural contexts work to create checklists (Chang, et al., 2015; Cullen et al., 2017; Yadav et al., 2019) of unprofessional behaviors to aid in the early identification and subsequent remediation of unprofessional behavior in medical students and residents.

Cluster 3 suggests that learning medical ethics is an important way for students and trainees to understand professionalism and the physician's role. Professionalism education through the formal curriculum of medical ethics has become a standard part of medical school curricula (O'Sullivan et al., 2012). A systematic review of teaching professionalism in postgraduate medical education also reveals that medical ethics in practice is one of the most common professionalism topics under discussion (Berger et al., 2020). However, we need to realize that it is not enough to rely solely on the medical ethics curriculum to teach professionalism, as more specialized professionalism education and integration of professionalism into the current curriculum would be needed. Endeavors to making professionalism a component of the medical curriculum and a measurable outcome that is valued by both students and trainees continue to pose a challenge for researchers and educators (O’Sullivan et al., 2012).

Having the lowest intra-cluster correlation and what is considered to be relatively peripheral development compared with clusters 1, 3, and 5, cluster 4 covers several subtopics related to healthcare professionals, such as knowledge, attitudes, and associated behaviors pertaining to health-related topics (Deniz et al., 2018; Sinclair et al., 2018), role in patient care teams, and interprofessional experiences in healthcare (Håkansson et al., 2019; Rydenfält et al., 2019). In this study, the target population on professionalism research mostly comprised physicians and medical students (Berger et al., 2020; Birden et al., 2013; Li et al., 2017). While research on other health personnel, such as nurses, pharmacists, and allied health professionals (e.g. community health workers, emergency medical technicians) may have been included as well, this clustering suggests professionalism research also should pay attention to these other professional subgroups (of which there are far fewer studies), especially their interprofessional relations, such as the collaboration between physicians, nurses, pharmacists, and hospital administrators in a patient care team in order to achieve high quality health care.

Finally, whether it is nurturing and assessing professionalism, or addressing professionalism lapses, the purpose of professionalism research and development is to focus on the primacy of patients' welfare and to improve the quality of health care, both of which would be inseparable from physician competency and practice pattern (ABIM Foundation et al., 2002). In addition, maintaining high levels of professionalism and proactive communication are important to improving patient satisfaction, building good doctor-patient relationships, and maintaining doctor-patient trust (Doukas \& Volpe, 2018; Petrocchi et al., 2019). 
This importance is affirmed by how Cluster 5 demonstrated the highest centrality and high inter-cluster correlations.

Our study had several limitations. First, for the purposes of this research, we chose to limit our initial search to the WOS database. Despite our comprehensive literature retrieval, future research may need to consider the characteristics of publications not included in the WOS database. Second, we did not include papers on professionalism published in 2020, and this is mainly because the databases (WOS and the MeSH database) update research continuously, and the collection and indexing of literature published in 2020 would take scientific databases an additional several months. We are also aware that the outbreak of COVID-19 in 2020 was a huge challenge for the medical profession and healthcare system as a whole, and therefore medical professionalism research hotspots may undergo new changes related to the pandemic. For future research, we suggest that research hotspots on professionalism in 2020 be compared with those outlined in our present study so as to explore the changes that may be affected by COVID-19. Third, when matching those papers included in the WOS database retrieval with their counterparts indexed in the PubMed database, 305 (6.55\%) papers could not be matched, which was due to the PubMed database's own collection scope. Although this contributes to a small degree of bias, we deemed that it would not affect our overall grasp of the research hotspots.

In summary, we conducted a bibliometric analysis of the external characteristics and research hotspots of publications on medical professionalism. Our reporting of quantifiable knowledge could help inform gaps in the field of medical professionalism and provide evidence and guidance for future work for researchers, physicians, and educators.

Supplementary Information The online version contains supplementary material available at https://doi. org/10.1007/s11192-021-03993-0.

Acknowledgements The authors would like to thank Professor Cui Lei (School of Medical Informatics, China Medical University) for his guidance on the bibliometric methods and his interpretations of the indicators of the mountain visualization map and strategic diagrams.

Authors' contributions W and XS designed the research. XS and HL collected the data. XS, NJ, HL, and ND performed the data analyses. XS and NJ wrote the manuscript. All authors have read and approved the final manuscript.

Funding This study was supported by the Natural Science Foundation of Liaoning Province of China (20180550272), and the Medical Education Program of China Medical University (CMU Teaching Grant [2016]4-3). The funders had no role in the study design, data collection and analysis, decision to publish, or preparation of this manuscript.

Data availability All data supporting the conclusions of this article are included within the article (and its additional files).

Declarations

Conflict of interest The authors declare no potential conflict of interest.

Ethics approval and consent to participate This was a retrospective bibliometric study that did not involve human subjects. Therefore, the need for institutional ethics review board approval was waived. 


\section{References}

Accreditation Council for Graduate Medical Education. (2004). ACGME Outcome Project: Advancing education in medical professionalism. http://www.acgme.org/outcome/implement/Profm_resource.pdf

ABIM Foundation, ACP-ASIM Foundation, European Federation of Internal Medicine. (2002). Medical professionalism in the new millennium: A physician charter. Annals of Internal Medicene, 136(3), 243-246. https://doi.org/10.7326/0003-4819-136-3-200202050-00012

American Board of Internal Medicine Committee on Evaluation of Clinical Competence. (1995). Project professionalism. Philadelphia, PA: ABIM.

Aylott, L. M. E., Tiffin, P. A., Saad, M., Llewellyn, A. R., \& Finn, G. M. (2019). Defining professionalism for mental health services: A rapid systematic review. Journal of Mental Health, 28(5), 546-565. https://doi.org/10.1080/09638237.2018.1521933.

Azer, S. A., \& Azer, S. (2019). Top-cited articles in medical professionalism: A bibliometric analysis versus altmetric scores. British Medical Journal Open, 9(7), e029433. https://doi.org/10.1136/bmjop en-2019-029433.

Aziz, A. B., \& Ali, S. K. (2020). Relationship between level of empathy during residency training and perception of professionalism climate. BMC Medical Education, 20(1), 320. https://doi.org/10.1186/ s12909-020-02231-0.

Barnhoorn, P. C., Houtlosser, M., Ottenhoff-de Jonge, M. W., Essers, G., Numans, M. E., \& Kramer, A. W. M. (2019). A practical framework for remediating unprofessional behavior and for developing professionalism competencies and a professional identity. Medical Teacher, 41(3), 303-308. https://doi.org/ 10.1080/0142159x.2018.1464133.

Berger, A. S., Niedra, E., Brooks, S. G., Ahmed, W. S., \& Ginsburg, S. (2020). Teaching professionalism in postgraduate medical education: A systematic review. Academic Medicine, 95(6), 938-946. https://doi. org/10.1097/acm.0000000000002987.

Birden, H., Glass, N., Wilson, I., Harrison, M., Usherwood, T., \& Nass, D. (2013). Teaching professionalism in medical education: a Best Evidence Medical Education (BEME) systematic review. BEME Guide No. 25. Medicle Teacher, 35(7), e1252-1266. https://doi.org/10.3109/0142159x.2013.789132.

Birden, H., Glass, N., Wilson, I., Harrison, M., Usherwood, T., \& Nass, D. (2014). Defining professionalism in medical education: A systematic review. Medical Teacher, 36(1), 47-61. https://doi.org/10.3109/ 0142159x.2014.850154.

Borgmann, H., Cooperberg, M., Murphy, D., Loeb, S., N’Dow, J., Ribal, M. J., \& Kutikov, A. (2018). Online Professionalism-2018 Update of European Association of Urology (@Uroweb) Recommendations on the appropriate use of social media. European Urology, 74(5), 644-650. https://doi.org/10. 1016/j.eururo.2018.08.022.

Brennan, N., Price, T., Archer, J., \& Brett, J. (2020). Remediating professionalism lapses in medical students and doctors: A systematic review. Medical Education, 54(3), 196-204. https://doi.org/10.1111/ medu.14016.

Callon, M., \& Laville, F. (1991). Co-word analysis as a tool for describing the network of interactions between basic and technological research: The case of polymer chemsitry. Scientometrics, 22(1), 155-205.

Callon, M., Law, J., \& Rip, A. (1986). Mapping the dynamics of science and technology: Sociology of science in the real world. . The Macmillan Press.

Chang, H. J., Lee, Y. M., Lee, Y. H., \& Kwon, H. J. (2015). Investigation of unethical and unprofessional behavior in Korean RESIDENCY TRAIning. Teaching and Learning in Medicine, 27(4), 370-378. https://doi.org/10.1080/10401334.2015.1077128.

Chen, C. M. (2003). Citespace: Visualizing patterns and trends in scientific literature. http://cluster.cis. drexel.edu/ cchen/citespace/

Chen, C. M. (2014). TheCiteSpaceManual. http://cluster.ischool.drexel.edu/ cchen/citespace/CiteSpaceM anual.pdf

Chinese Medical Doctor Association. (2014). Code of ethics for Chinese physicians. 2014. http://www. cmda.net/gzdt12/10408.jhtml.

Clarivate. (2020).Counting method for articles and citations. https://esi.help.clarivate.com/Content/esi-thres holds.htm

Clarivate. (2016). HistCite. 2016. http://www.histcite.com/

Clarivate. (2021). Web of science platform. https://clarivate.com/webofsciencegroup/solutions/webofscien ce-platform/

Cui, L., Yan, L., et al. (2008). Development of a text mining system based on the co-occurrence of bibliographic items in literature databases. Chinese Journal of Data Analysis and Knowledge Discovery, 8, 70-75. 
Cullen, M. J., Konia, M. R., Borman-Shoap, E. C., Braman, J. P., Tiryaki, E., Marcus-Blank, B., \& Andrews, J. S. (2017). Not all unprofessional behaviors are equal: The creation of a checklist of bad behaviors. Medical Teacher, 39(1), 85-91. https://doi.org/10.1080/0142159x.2016.1231917.

Dart, J., McCall, L., Ash, S., Blair, M., Twohig, C., \& Palermo, C. (2019). Toward a global definition of professionalism for nutrition and dietetics education: A systematic review of the literature. Journal of the Academy of Nutrition and Dietetics, 119(6), 957-971. https://doi.org/10.1016/j.jand.2019.01.007.

Deniz, S., Coskun, F., Oztas, D., \& Kurt, A. O. (2018). Knowledge, attitudes and behaviors of breast and cervical cancers and screenings of women working in primary health care services. Journal of Balkan Union of Oncology, 23(7), 44-52.

Diederich Healthcare. (2017). 2017 Medical malpractice payout analysis. http://www.diederichhealthcare. com/thestandard/2017-medical-malpractice-payout-analysis/

Diem, A., \& Wolter, S. (2013). The use of bibliometrics to measure research performance in education sciences. Research in Higher Education, 54(1), 86-114.

Doukas, D. J., \& Volpe, R. L. (2018). Why pull the arrow when you cannot see the target? Framing professionalism goals in medical education. Academic Medicine, 93(11), 1610-1612. https://doi.org/10. 1097/acm.0000000000002264.

Ellaway, R. H., Coral, J., Topps, D., \& Topps, M. (2015). Exploring digital professionalism. Medical Teacher, 37(9), 844-849. https://doi.org/10.3109/0142159x.2015.1044956.

Ellegaard, O., \& Wallin, J. A. (2015). The bibliometric analysis of scholarly production: How great is the impact? Scientometrics, 105(3), 1809-1831. https://doi.org/10.1007/s11192-015-1645-z.

Fatollahi, J. J., Colbert, J. A., Agarwal, P., Lee, J. L., Lehmann, E. Y., Yuan, N., \& Chretien, K. C. (2020). The impact of physician social media behavior on patient trust. AJOB Empirical Bioethics, 11(2), 77-82. https://doi.org/10.1080/23294515.2019.1678533.

Frank, J. R., Snell, L., \& Sherbino, J. (Eds.). (2015). CanMEDS 2015 physician competency framework. . Royal College of Physicians and Surgeons of Canada.

General Medical Council. (2013). Good medical practice. https://www.gmc-uk.org/ethical-guidance/ethic al-guidance-for-doctors

Gómez-Durán, E. L., Martin-Fumadó, C., \& Arimany-Manso, J. (2013). Legal medicine contributions to patient safety from ascertainment and evaluation to research in medical liability. International Journal of Legal Medicine, 127(5), 1051-1053. https://doi.org/10.1007/s00414-013-0885-9.

Håkansson Lindqvist, M., Gustafsson, M., \& Gallego, G. (2019). Exploring physicians, nurses and wardbased pharmacists working relationships in a Swedish inpatient setting: A mixed methods study. International Journal of Clinical Pharmacy, 41(3), 728-733. https://doi.org/10.1007/s11096-019-00812-8.

Hodges, B., Paul, R., Ginsburg, S., \& The Ottawa Consensus Group, M. (2019). Assessment of professionalism: From where have we come-to where are we going? An update from the Ottawa Consensus Group on the assessment of professionalism. Medical Teaching, 41(3), 249-255. https://doi.org/10. 1080/0142159x.2018.1543862

Hodges, B. D., Ginsburg, S., Cruess, R., Cruess, S., Delport, R., Hafferty, F., \& Wade, W. (2011). Assessment of professionalism: Recommendations from the Ottawa 2010 conference. Medical Teacher, 33(5), 354-363. https://doi.org/10.3109/0142159x.2011.577300.

Irby, D. M., \& Hamstra, S. J. (2016). Parting the clouds: Three professionalism frameworks in medical education. Academic Medicine, 91(12), 1606-1611. https://doi.org/10.1097/acm.0000000000001190.

Kravet, S., Bhatnagar, M., Dwyer, M., Kjaer, K., Evanko, J., \& Singh, H. (2019). Prioritizing patient safety efforts in office practice settings. Journal of Patient Safety, 15(4), e98-e101. https://doi.org/10.1097/ pts.0000000000000652.

Kumar Ghosh, S., \& Kumar, A. (2019). Building professionalism in human dissection room as a component of hidden curriculum delivery: A systematic review of good practices. Anatomical Sciences Education, 12(2), 210-221. https://doi.org/10.1002/ase.1836.

Law, J., Bauin, S., Courtial, J., \& Whittaker, J. (1988). Policy and the mapping of scientific change: A coword analysis of research into environmental acidification. Scientometrics, 14(3-4), 251-264.

Lee, K., Whelan, J. S., Tannery, N. H., Kanter, S. L., \& Peters, A. S. (2013). 50 years of publication in the field of medical education. Medical Teacher, 35(7), 591-598. https://doi.org/10.3109/0142159x.2013. 786168.

Lesser, C. S., Lucey, C. R., Egener, B., Braddock, C. H., 3rd., Linas, S. L., \& Levinson, W. (2010). A behavioral and systems view of professionalism. JAMA, 304(24), 2732-2737. https://doi.org/10.1001/jama. 2010.1864.

Li, H., Ding, N., Zhang, Y., Liu, Y., \& Wen, D. (2017). Assessing medical professionalism: A systematic review of instruments and their measurement properties. PLOS ONE, 12(5), e0177321. https://doi.org/ 10.1371/journal.pone.0177321. 
Linnenluecke, M. K., \& Singh, A. K. (2019). Conducting systematic literature reviews and bibliometric analyses. Australian Journal of Management, 45(2), 175-194.

Madaus, G. F., Scriven, M. S., \& Stufflebeam, D. L. (1983). Evaluation models: Viewpoints on educational and human services evaluation. . Kluwer-Nijhoff Publishing.

Michael, J. K. (2010). Usage bibliometrics. Annual Review of Information Science \& Technology, 44(1), 3.

Mueller, P. S. (2009). Incorporating professionalism into medical education: The Mayo Clinic experience. Keio Journal of Medicine, 58(3), 133-143. https://doi.org/10.2302/kjm.58.133.

National Center for Biotechnology Information, U.S. National Library of Medicine. (1974). Malpractice. 1974. https://www.ncbi.nlm.nih.gov/mesh/?term=Malpractice

National Center for Biotechnology Information, U.S. National Library of Medicine. (2002). Professional Misconduct. https://www.ncbi.nlm.nih.gov/mesh/?term=Professional+misconduct

National Center for Biotechnology Information, U.S. National Library of Medicine. (2016). Professionalism. https://www.ncbi.nlm.nih.gov/mesh/?term=professionalism

O’Sullivan, H., van Mook, W., Fewtrell, R., \& Wass, V. (2012). Integrating professionalism into the curriculum: AMEE Guide No. 61. Medical Teaching, 34(2), 64-77. https://doi.org/10.3109/0142159x.2012. 655610.

Ong, Y. T., Kow, C. S., Teo, Y. H., Tan, L. H. E., Abdurrahman, A., Quek, N. W. S., \& Radha Krishna, L. K. (2020). Nurturing professionalism in medical schools. A systematic scoping review of training curricula between 1990-2019. Medical Teaching, 42(6), 636-649. https://doi.org/10.1080/0142159x. 2020.1724921.

Pao, M. L. (2014). Automatic text analysis based on transition phenomena of word occurrences. Journal of the American Society for Information Science, 29(3), 121-124.

Petrocchi, S., Iannello, P., Lecciso, F., Levante, A., Antonietti, A., \& Schulz, P. J. (2019). Interpersonal trust in doctor-patient relation: Evidence from dyadic analysis and association with quality of dyadic communication. Social Science and Medicine, 235, 112391. https://doi.org/10.1016/j.socscimed.2019. 112391.

Pritchard, A. (1969). Statistical bibliography or bibliometrics? Journal of Documentation, 25(4), 348-349.

Rasmussen, M., Karypis, G. (2004). gCLUTO: An interactive clustering, visualization, and analysis system. http://glaros.dtc.umn.edu/gkhome/cluto/gcluto/publications

Rydenfält, C., Borell, J., \& Erlingsdottir, G. (2019). What do doctors mean when they talk about teamwork? Possible implications for interprofessional care. Journal of Interprofessional Care, 33(6), 714-723. https://doi.org/10.1080/13561820.2018.1538943.

School of Medical Informatics of China Medical University. (2008). Bibliographic item co-occurrence matrix builder. https://www.cmu.edu.cn/dmi/nr.jsp?urltype=news.NewsContentUrl\&wbtreeid=2161\& wbnewsid $=4589$ \&archive $=0$

Sinclair, S., Hack, T. F., Raffin-Bouchal, S., McClement, S., Stajduhar, K., Singh, P., \& Chochinov, H. M. (2018). What are healthcare providers' understandings and experiences of compassion? The healthcare compassion model: A grounded theory study of healthcare providers in Canada. British Medical Journal Open, 8(3), e019701. https://doi.org/10.1136/bmjopen-2017-019701.

Tscharntke, T., Hochberg, M. E., Rand, T. A., Resh, V. H., \& Krauss, J. (2007). Author sequence and credit for contributions in multiauthored publications. PLoS Biology, 5(1), e18. https://doi.org/10.1371/journ al.pbio.0050018.

US Department of Health and Human Services. (2017). NPDB research statistics. https://www.npdb.hrsa. gov/resources/npdbstats/npdbStatistics.jsp\#ContentTop

Yadav, H., Jegasothy, R., Ramakrishnappa, S., Mohanraj, J., \& Senan, P. (2019). Unethical behavior and professionalism among medical students in a private medical university in Malaysia. BMC Medical Education, 19(1), 218. https://doi.org/10.1186/s12909-019-1662-3. 